\title{
Personalized Modeling Pipeline for Cardiac Electrophysiology Simulations of Cardiac Resynchronization Therapy in Infarct Patients
}

\author{
Caroline Mendonca Costa ${ }^{1}$, Aurel Neic ${ }^{2}$, Gernot Plank ${ }^{2}$, Eric Kerfoot ${ }^{1}$, Bradley Porter ${ }^{1}$, Benjamin \\ Sieniewicz $^{1}$, Justin Gould ${ }^{1}$, Baldeep Sidhu ${ }^{1}$, Zhong Chen ${ }^{1}$, Christopher A Rinaldi ${ }^{3}$, Martin J Bishop ${ }^{1}$, \\ Steven A Niederer ${ }^{1}$ \\ ${ }^{1}$ King's College London, UK \\ ${ }^{2}$ Medical University of Graz, Austria \\ ${ }^{3}$ Guy's and St. Thomas' NHS Foundation Trust, UK
}

\begin{abstract}
Cardiac Resynchronization Therapy (CRT) is associated with increased arrhythmogenic risk in infarct patients when pacing adjacent to a scar. We investigated the role of pacing location relative to scar on dispersion of repolarization, as a surrogate for arrhythmogenic risk. For this task, we developed a personalization and simulation pipeline that allows fast development of personalized computational models and simulation of cardiac electrophysiology. Twenty four models of left ventricular anatomy and scar morphology were built and repolarization sequences were simulated. Simulation results show that CRT increases dispersion of repolarization around a scar when pacing adjacent to it, thus, providing a mechanistic explanation of increased arrhythmogenic risk in infarct patients undergoing CRT.
\end{abstract}

\section{Introduction}

Cardiac Resynchronization Therapy (CRT) is an effective treatment for heart failure. However, there is evidence that CRT is arrhythmogenic in infarct patients. Particularly, pacing adjacent to a scar is associated with increased risk of ventricular tachycardia (VT) [1]. However, the underlying mechanisms of increased VT risk are not known.

CRT alters the heart's activation and repolarization sequences and it has been associated with increased dispersion of repolarization, a known risk factor for re-entry [2]. Thus, we hypothesize that CRT increases dispersion of repolarization in infarct patients when pacing adjacent to a scar, which explains their increased VT risk.

Investigating the role of pacing location during CRT on VT risk in the clinical setting is hampered by the anatomy of the coronary sinus, which limits where CRT pacing leads can be implanted. Computational models provide full flexibility, thus, allowing a detailed and sys- tematic investigation of the role of pacing location. However, building clinically relevant models, that include realistic anatomy and scar morphology, and simulating cardiac electrophysiology (EP) at clinical time-scales remains a challenging task. Therefore, we developed a personalization pipeline, which allows fast development of infarct model cohorts and EP simulations.

The developed pipeline was used to build 24 personalized models of left ventricular anatomy and scar morphology, which were then used to investigate the role of pacing location on dispersion of repolarization, as a surrogate for VT risk. Our simulation results show that pacing adjacent to a scar increases dispersion of repolarization in its vicinity, which provides a mechanistic explanation for increased VT risk in CRT patients with infarct.

\section{Methods}

\subsection{Patient cohort}

Twenty four patients with ischemic heart failure undergoing CRT were included in our study. Details on patient characteristics can be found in [3].

Cardiac magnetic resonance imaging was performed using a 1.5-T scanner with a 32-channel coil (Philips Healthcare, Best, The Netherlands). A stack of short-axis slices was acquired $10-15$ minutes after contrast injection (gadobutrol $0.2 \mathrm{mmol} / \mathrm{kg}$ body weight) for late gadolinium enhancement magnetic resonance imaging (LGE-MRI). Further details on the imaging protocols can be found in [3]. In-plane resolution varied from $0.6 \times 0.6 \mathrm{~mm}$ to 1.37 $\times 1.37 \mathrm{~mm}$. Slice thickness varied from 8 to $20 \mathrm{~mm}$.

\subsection{Construction of personalized anatomi- cal models}

We developed a pipeline for fast construction of personalized anatomical models. The methods used are described 
in the following sub-sections. A schematic of the pipeline is shown in Figure 1.

\subsubsection{Image segmentation and processing}

Left ventricular (LV) endocardium and epicardium contours were manually drawn in each short-axis slice using the image segmentation software Eidolon [4]. The infarct scar and border zone (BZ) were segmented as the regions with signal intensity above 3 and 2 standard deviations from the mean signal intensity within healthy myocardium, respectively, as described previously [3]. The 2D scar and BZ segmentations were reconstructed in 3D using a statistical method for shape reconstruction based on the logarithm of odds function, as described previously [5].

\subsubsection{Model generation}

A finite element mesh of tetrahedral elements was generated by interpolating the LV endocardium and epicardium contours using Eidolon [4]. This mesh was then refined using the C-GAL library [6] to achieve a mean edge length of $0.8 \mathrm{~mm}$. The 3D reconstructed scar and $\mathrm{BZ}$ segmentations were mapped onto the tetrahedral mesh and used to label mesh elements as scar, BZ, and healthy tissue. Fiber orientations were assigned to each anatomical model using a Laplace-Dirichlet rule-based algorithm [7].

\subsection{Simulation setup}

We performed simulations of cardiac EP using our models of personalized anatomy and the Cardiac Arrhythmia Research Package (CARP) $[8,9]$ to investigate the role of $\mathrm{LV}$ epicardial pacing location relative to scar in dispersion of repolarization. The mathematical model and parameters used, pacing locations, and computed metrics are described in the following sections.

\subsubsection{Electrophysiology models}

Propagation of cardiac action potentials was simulated using a Reaction-Eikonal model [10], which allows the use of coarser spatial resolutions than the cardiac monodomain model, thus, reducing computational cost. This model was coupled with the Ten Tusscher model for the action potential of human ventricular cells [11]. No difference in ionic currents between endocardium and epicardium cells was considered in this study.

\subsubsection{Model parameters}

Transversely isotropic conduction velocities of 0.67 and $0.3 \mathrm{~m} / \mathrm{s}$ in the longitudinal and transverse directions, respectively, were assigned to healthy tissue. The BZ was

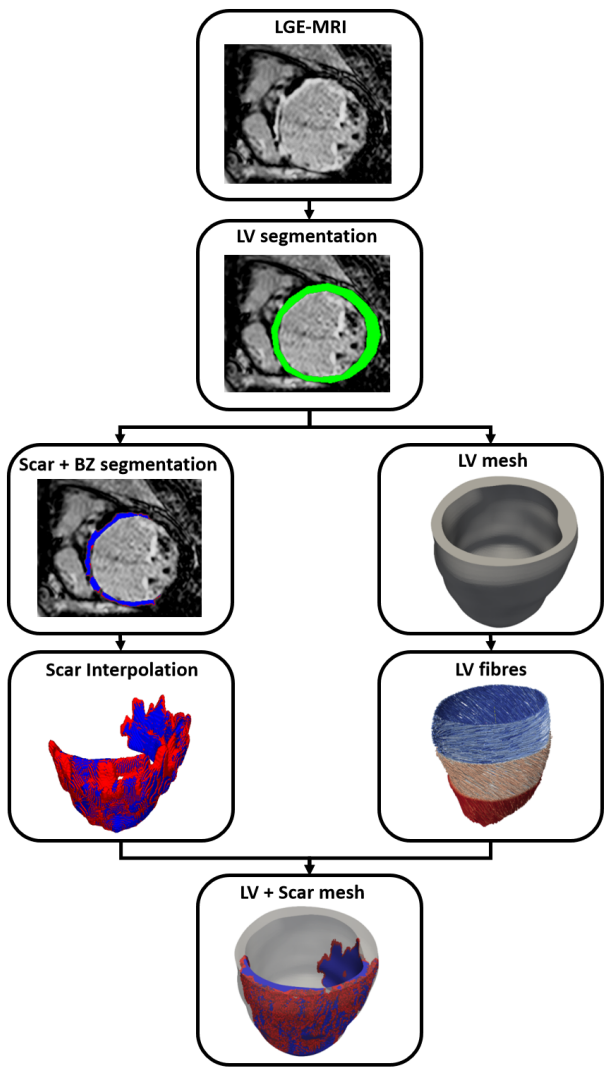

Figure 1. Pipeline for construction of models of personalized anatomy. From left to right: the left ventricular (LV) wall is manually segmented; the scar and border zone (BZ) thresholded; an LV tetrahedral mesh is generated and rulebased fibers are assigned; the 2D scar and BZ segmentations are interpolated to $3 \mathrm{D}$ and merged with the tetrahedral mesh to label mesh elements as healthy, scar, and BZ.

assigned isotropic conduction velocity of $0.15 \mathrm{~m} / \mathrm{s}$, corresponding to $50 \%$ of the transverse velocity in healthy tissue, according to experimentally measured values $[12,13]$. The scar core was modeled as unexcitable non-conducting tissue, assuming it consists of predominantly collagenous non-conducting material [14].

\subsubsection{Pacing locations}

Pacing locations at 0.5 and $4.5 \mathrm{~cm}$ from the scar surface were chosen for each model. Distances from scar were computed using eikonal-based activation maps [10], where activation was initiated at all vertices on the scar surface and the core of scar was set as non-conducting. The conduction velocity was set to $1 \mathrm{~m} / \mathrm{s}$, thus giving activation time equal distance. 


\subsubsection{Repolarization sequences and metrics}

One paced beat was simulated for 1 second. Repolarization times were computed as the instant where the transmembrane voltage reached a threshold of $-70 \mathrm{~ms}$ following activation. Local dispersion of repolarization was computed as the magnitude of the repolarization gradient at each mesh point.

Experimental [15] and simulation [16] studies have demonstrated that a repolarization gradient of $3 \mathrm{~ms} / \mathrm{mm}$ can lead to uni-directional block, a pre-requisite for reentry inducibility. Assuming that the larger the volume of tissue with high gradients, the higher the probabity of uni-directional block, we computed the volume of high repolarization gradients (HRG), which are above $3 \mathrm{~ms} / \mathrm{mm}$, as a metric of dispersion of repolarization and a surrogate for VT risk.

\section{Results}

\subsection{Personalized anatomical models}

Two examples of the resulting $24 \mathrm{LV}$ models are shown in Figure 2. Healthy tissue is shown in gray, whereas scar core and BZ are shown in blue and red, respectively. Each model was generated on a desktop workstation with 6 cores and 64GB of RAM in less than 2 hours.
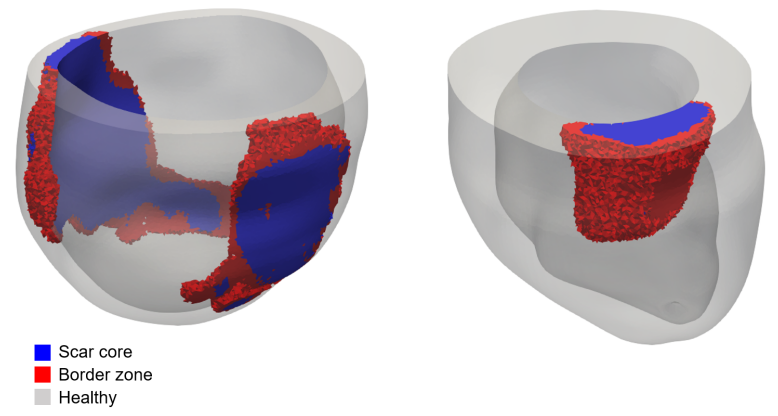

Figure 2. Personalized models of left ventricular anatomy and scar morphology. Healthy tissue is displayed in gray, scar core in blue, and border zone in red.

\subsection{Effect of pacing location on repolariza- tion}

Repolarization sequences where simulated for each model in $\approx 14$ minutes using 32 cores of a large shared memory system consisting of 640 cores and 5TB of RAM.

An example of the spatial distribution of repolarization gradients within the LV is shown in Figure 3-A). High repolarization gradients (HRG) appear in the border zone and around the LV lead. The volume of HRG around the scar is larger when pacing near instead of away from it. A boxplot of the volumes of HRG $(1 \mathrm{~cm})$ around the scar when pacing 0.5 and $4.5 \mathrm{~cm}$ from the scar for all models are shown in Figure 3-B). The volumes are significantly (P $<0.000001)$ larger when pacing near the scar than away from it.
A)
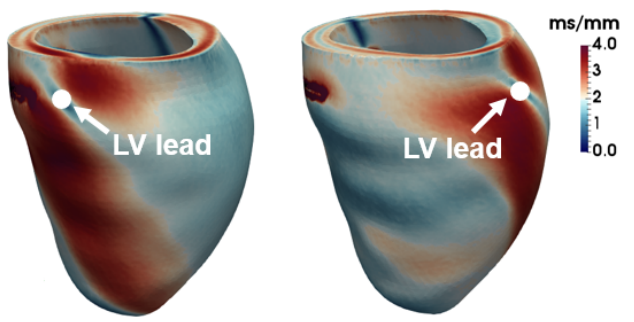

B)
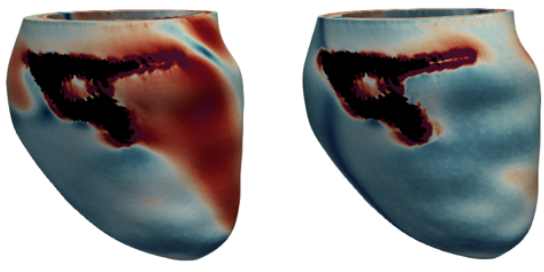

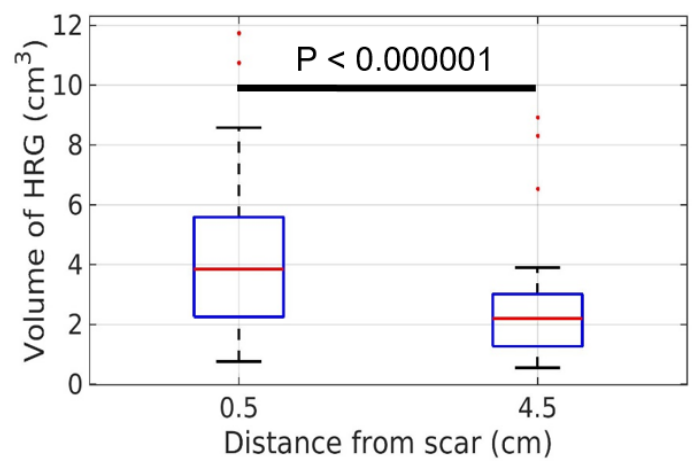

Figure 3. A) Spatial distribution of repolarization gradients when pacing $0.5 \mathrm{~cm}$ (left panes) and $4.5 \mathrm{~cm}$ from scar (right panes). B) Comparison between the volume of high repolarization gradients (HRG) around the scar when pacing 0.5 and $4.5 \mathrm{~cm}$ from the scar.

\section{Discussion}

The personalization pipeline developed in this study allows fast development of model cohorts while employing the Reaction-Eikonal model allows running EP simulations at a time-scale compatible with the clinical setting, thus, facilitating clinical translation of computational modeling.

Re-entrant arrhythmias in patients with infarct typically originate at the scar, particularly at the BZ, where ectopic foci are more likely to occur [17]. The high repolarization gradients observed around the scar when pacing adjacent 
to it, as shown by our simulations, may be sufficient to cause uni-directional conduction block of a nearby ectopic focus and lead to re-entry $[2,18]$. Thus, increased volume of high repolarization gradients around a scar when pacing adjacent to it provides a plausible mechanistic explanation for increased VT risk in infarct patients undergoing CRT and suggests that pacing away from a scar may mitigate VT risk in these patients.

\section{Acknowledgements}

The research was funded/supported by the National Institute for Health Research Biomedical Research Centre and CRF based at Guy's and St Thomas'NHS Foundation Trust and King's College London (KCL), by the Wellcome EPSRC Centre for Medical Engineering at KCL (WT 203148/Z/16/Z), by the Medical Research Council (MR/N011007/1), and by the British Heart Foundation (RE/08/003, PG/15/91/31812, and PG/16/81/32441).

\section{References}

[1] Roque C, Trevisi N, Silberbauer J, Oloriz T, Mizuno H, Baratto F, Bisceglia C, Sora N, Marzi A, Radinovic A, Guarracini F, Vergara P, Sala S, Paglino G, Gulletta S, Mazzone P, Cireddu M, Maccabelli G, Della Bella P. Electrical storm induced by cardiac resynchronization therapy is determined by pacing on epicardial scar and can be successfully managed by catheter ablation. Circulation Arrhythmia and Electrophysiology 2014;7(6):1064-1069.

[2] Clayton RH, Holden AV. Dispersion of cardiac action potential duration and the initiation of re-entry: a computational study. Biomedical Engineering online 2005;4:11.

[3] Chen Z, Sohal M, Voigt T, Sammut E, Tabon C, Child N, Jackson T, Shetty A, Bostock J, Cooklin M, Wright M, Murgatroyd F, Gill J, Carr-White G, Chiribiri A, Schaeffter Ph TD, Razavi R, Aldo Rinaldi C, Schaeffter T. Myocardial tissue characterization by cardiac magnetic resonance imaging using $\mathrm{t} 1$ mapping predicts ventricular arrhythmia in ischemic and non-ischemic cardiomyopathy patients with implantable cardio- verter defibrillators. Heart Rhythm 2014;12:792-801.

[4] Kerfoot E, Fovarge L, Rivolo S, Shi W, Rueckert D, Nordsletten D, Lee J, Chabiniok R, Razavi R. Eidolon: visualization and computational framework for multi-modal biomedical data analysis. In LNCS 9805, Medical Imaging and Augmented Reality 2016 (MIAR 2016), volume 9805. Springer, Aug 2016; 425-437.

[5] Ukwatta E, Rajchl M, White J, Pashakhanloo F, Herzka DA, McVeigh E, Lardo AC, Trayanova N, Vadakkumpadan F. Image-based reconstruction of 3D myocardial infarct geometry for patient specific applications. Proceedings of SPIE the International Society for Optical Engineering 2015;73(4):94132W.
[6] The CGAL Project. CGAL User and Reference Manual. 4.12.1 edition. CGAL Editorial Board, 2018. URL https://doc.cgal.org/4.12.1/Manual/ packages.html.

[7] Bayer JD, Blake RC, Plank G, Trayanova NA. A novel rulebased algorithm for assigning myocardial fiber orientation to computational heart models. Annals of Biomedical Engineering 2012;40(10):2243-2254.

[8] Vigmond EJ, Hughes M, Plank G, Leon LJ. Computational tools for modeling electrical activity in cardiac tissue. Journal of Electrocardiology 2003;36(SUPPL.):69-74.

[9] Vigmond EJ, Weber dos Santos R, Prassl AJ, Deo M, Plank G. Solvers for the cardiac bidomain equations. Progress in Biophysics and Molecular Biology 2008;96(1-3):3-18.

[10] Neic A, Campos FO, Prassl AJ, Niederer SA, Bishop MJ, Vigmond EJ, Plank G. Efficient computation of electrograms and ECGs in human whole heart simulations using a reaction-eikonal model. Journal of Computational Physics 2017;346(346):191-211.

[11] Ten Tusscher KHWJ, Panfilov AV. Alternans and spiral breakup in a human ventricular tissue model. Am J Physiol Heart Circ Physiol 2006;291:1088-1100.

[12] Mendonca Costa C, Plank G, Rinaldi CA, Niederer SA, Bishop MJ. Modeling the electrophysiological properties of the infarct border zone. Frontiers in Physiology 2018;9.

[13] Ursell PC, Gardner PI, Albala A, Fenoglio JJ, Wit AL. Structural and electrophysiological changes in the epicardial border zone of canine myocardial infarcts during Infarct Healing. Circ Res 1985;

[14] Rutherford SL, Trew ML, Sands GB, Legrice IJ, Smaill BH. High-resolution 3-dimensional reconstruction of the infarct border zone: Impact of structural remodeling on electrical activation. Circulation Research 2012;111(3):301-311.

[15] Laurita KR, Rosenbaum DS. Interdependence of modulated dispersion and tissue structure in the mechanism of unidirectional block. Circulation research 2000;87(10):922-928.

[16] Qu Z, Garfinkel A, Weiss JN. Vulnerable window for conduction block in a one-dimensional cable of cardiac cells, 1 : single extrasystoles. Biophysical Journal 2006;91(3):793804.

[17] Bakker JM, Capelle FJ, Janse MJ, Wilde AA, Coronel $\mathrm{R}$, Becker AE, Dingemans KP, Hemel NM, Hauer RN. Reentry as a cause of ventricular tachycardia in patients with chronic ischemic heart disease: electrophysiologic and anatomic correlation. Circulation 1988;77(84076).

[18] Kleber AG, Rudy Y. Basic Mechanisms of Cardiac Impulse Propagation and Associated Arrhythmias. Physiological Reviews 2004;84(2):431-488.

Address for correspondence:

Caroline Mendonca Costa

School of Biomedical Engineering and Imaging Sciences

King's College London, United Kingdom

caroline.mendonca_costa@kcl.ac.uk 